\title{
A Framework for Metadata Creation Tools
}

\author{
Valentina Malaxa \\ DuvaSawko, Inc. \\ Daytona, Florida, USA
}

malaxa@acm.org

\author{
lan Douglas \\ Florida State University \\ Tallahassee, Florida, USA
}

idouglas@Isi.fsu.edu

\begin{abstract}
Metadata is an increasingly important aspect of resource discovery. Good metadata has the potential to increase discovery and reuse and to facilitate interoperability of digital assets. Using the domain of learning objects, issues associated with the application of metadata standards and the challenges in metadata creation are examined. A framework for customizable metadata authoring that addresses the issues and challenges is described. The framework consists of flexible metadata schema, metadata schema views, templates, collaborative metadata editing, contextual help, and an effective interface component selection. A prototype implementation of the framework, CLOMAT (Customizable Learning Objects Metadata Authoring Tool), is used to illustrate the framework in operation. An initial evaluation of this prototype indicates substantial productivity gains over conventional metadata creation tools.
\end{abstract}

Keywords: Metadata, learning objects, editing, reuse

\section{Introduction}

Discovery and reuse of digital assets, particularly non-textual assets, benefits from the availability of human-created metadata. The main challenges to human-created metadata noted in the literature is the potentially high cost of production in terms of human effort, time, money, and the errors and inconsistency that occur (Geisler, McArthur, \& McClelland, 2002; Marshall, 1998). The overhead involved in creating and managing metadata is a potential barrier to the successful use of metadata to facilitate reuse and sharing. Organizations require efficient mechanisms for reducing the overhead and should be able to customize their metadata creation according to changing standards and their internal needs.

Metadata, generally defined as structured data about data, is helpful to the efficient discovery and reuse of digital assets. In the case of learning objects, metadata describes to the outside world the purpose the object serves, the way to access and activate the object, and the way to use it in the context of the desired knowledge. A learning object is generally defined as a digital resource con-

Material published as part of this journal, either on-line or in print, is copyrighted by the publisher of the Informing Science Journal. Permission to make digital or paper copy of part or all of these works for personal or classroom use is granted without fee provided that the copies are not made or distributed for profit or commercial advantage AND that copies 1) bear this notice in full and 2) give the full citation on the first page. It is permissible to abstract these works so long as credit is given. To copy in all other cases or to republish or to post on a server or to redistribute to lists requires specific permission and payment of a fee. Contact Publisher@ijklo.org to request redistribution permission. taining content to facilitate learning. A learning object implies a certain degree of granularity, so thus an online course may be made up of a large number of objects that are individually stored in a learning repository. The storage of reusable digital assets in repositories is a common goal that metadata promises to facilitate. Frameworks are being developed to provide interoperability for digital 
libraries (Arms et al., 2002), with many using the protocol for metadata harvesting developed by the Open Archives Initiative (Lagoze \& Van de Sompel, 2001). The first accepted metadata standard for learning objects was IEEE Learning Object Metadata (Learning Objects Metadata [LOM], 2002). The purpose of this standard is to facilitate search, evaluation, acquisition, and use of learning objects, by learners or instructors, for instance. The LOM standard is based upon the Dublin Core Metadata Abstract Model (2005), the first internationally established online metadata model that supported a broad range of purposes.

\section{The Metadata Problem}

Defining a standard for metadata is a necessary first step towards facilitating discovery and reuse. In addition to standards, attention should be paid to the way the metadata is created, as this will affect the discoverability of the data to which it refers.

\section{Economics of Metadata Creation}

There is a trade-off to be considered in metadata creation. The greater the amount of metadata attached to an object, the higher the potential for discoverability; however, there is a higher cost in producing that metadata. South and Monson (2000) report a physical science online course that consisted of 34 lessons and approximately 350 web pages, containing over 1300 media objects, ranging from simulations to charts and diagrams. When tracking so many digital objects, the cost of creating high quality metadata for each object, as well as the cost of storing and managing them becomes a significant issue. A number of studies demonstrate that entering complex metadata efficiently, accurately, and consistently can be confusing, costly, time consuming and errorprone (Greer, 2002; Kunze, Brase, \& Nejdl, 2002; Marshall, 1998). Inconsistencies in metadata assigned to resources can arise due to variations in a given cataloger's judgment over time and because different catalogers may make varied judgments in cataloging resources. Another challenge was that metadata standards often required adjustments based on the particular collection and its use (Geisler et al., 2002, Marshall, 1998).

\section{Continual Evolution of Standards}

Kunze, Brase, and Nejdl (2002) report that most editors or viewers for metadata learning resources that use the LOM standard concentrate only on the current standard and lack flexible structure to adapt to new standards. Also, they believe that the LOM standard, regardless of the very useful work that has been done in developing it, still has space for improvement in specifying important educational aspects of learning resources. For example, Poyry Pelto-Aho, and Puustjarvi (2002) report on a virtual university project (CUBER) that required the development of an extension to the LOM standard. This suggests the need for flexible learning object metadata tools that are able to adapt to future metadata models or current extensions. The specialized metadata requirements for video and picture metadata authoring should be noted (Ryu, Sohn, \& Kim, 2002; Yao \& Jin, 2001), given that the raw video and images do not possess the same user-level information as text and thus are not directly searchable or reusable in the same way. Saddik, Ghavam, Fischer, \& Steinmetz (2000) also report difficulties with current standards in relation to dynamic digital objects such as video and simulations. This work suggests there is a need to facilitate different perspectives that allow the creation of metadata for different purposes.

\section{Specialized Knowledge Requirement}

Due to the large number of learning objects that must be tagged with metadata, people who are not metadata experts will become involved in the metadata authoring process. Those people must also be provided with effective just-in-time support. To provide more insight into the user experience while implementing metadata standards, the Center for Educational Technology Interopera- 
bility Standards (CETIS), in the United Kingdom, developed case studies. The aim of producing these case studies was to raise awareness of how and why people are implementing educational metadata specifications and to provide some reflection on what problems were encountered and what worked well. In one case, Barker and Ryan (2005) found that several metadata elements caused some difficulties, given the catalogers were not the creators of the resources being tagged. In particular, the technical requirements and educational description required specialist knowledge and care in maintaining consistency between entries from different catalogers. Educational descriptions were considered to be subjective, and many users considered the URL and general description to be completely sufficient for their purposes.

In a second case, there was difficulty in making sure the authors understood the purpose of the metadata and the method used to enter it, and there were not enough best practice guidelines available. The elements with definite values, such as educational difficulty, were considered to be more useful than descriptive elements, which were thought to be too subjective. Regardless of the skill level of metadata creators, whether experts or not, the interface plays an important role in any computer-related endeavor. Effective functionality with a poorly designed interface will remain unusable and unused.

\section{A Framework for Metadata Creation Tools}

Based on the review of studies presented in the previous section and a review of existing editors for metadata (e.g. Candan, Liu, \& Suvarna, 2001; Grissom et al., 1998; Lagoze \& Van de Sompel, 2001; Marshall, 1998), a framework for the creation of optimal tools for metadata creation was determined to include the following features.

\section{Flexible Metadata Schema}

The possibility for a flexible metadata schema incorporated into a metadata authoring tool would help those tools become "future-proof," and adaptable to evolving standards and higher user expectations.

\section{Metadata Schema Views}

The possibility for multiple views of the same metadata schema would allow metadata creators to select and display only those metadata elements necessary for the description of the learning objects for their particular context or project. Thus, metadata creators need not be limited by a strictly defined schema and need not "reinvent the wheel" by building an entirely new metadata schema.

\section{Metadata Templates}

Kassanke and Steinacker (2000) noted that when tagging the source material with the LOM editor (Marshall, 1998), most elements of a lesson to be described apply the same basic metadata information, such as the name of the author, the rights of the lesson, or the targeted user group. From this, it was concluded that templates were needed to increase the efficiency of metadata creation. A separate study by Saddik, Ghavam, Fischer and Steinmetz (2000) came to the same conclusion.

Metadata templates represent a potential solution for the challenge of creating metadata for significantly higher numbers of learning objects. Metadata creators can use templates to store personal profile information (e.g. name, type of contribution) or specific information related to the learning objects (e.g. keywords, media type, level of granularity). Thus, they do not have to reenter the same information for each object. Creating a template with $m$ metadata elements already edited for a group of $n$ learning objects reduces the cost of editing the metadata for those learning objects by $m^{*}(n-1)$ times. If the grouping of the learning objects is optimal and $\mathrm{m}$ approaches the 
total number of metadata elements that schema has, the time for editing $n$ learning objects approaches the time for editing one learning object.

\section{Collaborative Metadata Editing}

An editor which allows editing content online from anywhere in the world without additional client software, plug-ins, or configuration represents a possibility for reducing the workload for metadata creators. For example, the subject matter expert can provide the design specification for a new learning object, the instructional designer can edit those metadata elements related to educational aspects of the learning objects, and the technical staff can take care of technical details (e.g. the software needed to access the learning resource or the size in bytes of the resource). In this way, the metadata can reach a higher level of richness, completeness, and accuracy.

\section{Contextual Help}

Novice metadata creators often face many difficulties in mastering current metadata editing systems. Contextual help provides immediate assistance to users without their having to leave the context in which they are working. Contextual help allows the process and the concepts to be explained while the user executes a relevant task. Also, contextual help supports the user activity and is available according to the task they perform, enabling a better assimilation of the concepts or processes from the application they use (Garcia, 2000).

\section{Effective Interface}

Visual representations of metadata schemas, drop-down lists, controlled vocabularies, and fill-inthe-blank fields must be part of an effective interface that will allow the management of all kinds of metadata elements (e.g. simple, composed, range, multi-entry) in an organized way. The design techniques have to be based on global usability principles and guidelines (e.g. ease of learning, flexibility, familiarity, consistency, predictability, recoverability). For example, a poor layout and design of a form (one large form) can be avoided by breaking up the form into digestible, self-explanatory segments of information, which will provide the user with the adequate feedback for the state of the system while the task is gradually being accomplished.

\section{Prototype Implementation}

A proof-of-concept prototype was created in order to test the efficiency gains achievable by an editor conforming to the framework. Customizable Learning Objects Metadata Authoring Tool (CLOMAT) is a browser-based metadata editor which uses standard (e.g. SCORM model) and self-defined metadata schemas to represent the structure and meta-information of different learning objects. An SQL Database Server is used to store the metadata schemas and metadata elements' values. The metadata values can be exported to an XML file that can be packaged with the learning object and stored in learning object repositories. The CLOMAT prototype demonstrates several techniques to overcome some of the main difficulties of authoring in this domain; points a user-centered way to a future in which everyone can be as comfortable editing metadata as they are today editing text; intends to make metadata editing easier, complete and accurate; and makes use of the latest standards without being fixed to them.

\section{The Tree Structure of Metadata}

The use of a hierarchical decomposition of a classification problem allows for efficiencies in both learning and representation because each sub-problem is smaller than the original problem (Dumais \& Chen, 2000). Most of the metadata model definitions, such as LOM, are hierarchies (see Figure 1). 
Since hierarchical models are convenient for representing metadata consisting of many elements and sub-elements, and they also are very useful to navigate such collections of elements, the design decision to represent metadata models in a tree view structure was made. This was considered to simplify the mental models users must build to understand the metadata conceptual model.

\section{The Meta-Metadata Model}

Meta-metadata is an abstraction of metadata just as metadata is an abstraction of the data. The meta-metadata model is seen in the present paper as a means of facilitating the representation and extensibility of a metadata model (see Figure 2). Standards are developed as means to an end, and metadata standards cannot work very well without a great deal of flexibility. One size cannot possibly fit all, and the developers of the metadata standards recognize this. The elements in the standards may determine what the creators of metadata can say about an object, but it is the creators' choice to decide how, how often, and in how many different ways they say it. For example, metadata creators can specify an English title, a French title, and a Chinese title for the same learning content; they can add individualized lists of keywords for classifying instructional material; and they can refine an element like contributor so that they can better differentiate among types of contributors (e.g. primary versus secondary contributors). To date, various standards bodies have been concerned primarily with the metadata elements, which is why there are today large standards with 80 metadata elements. There has not been as much emphasis placed on

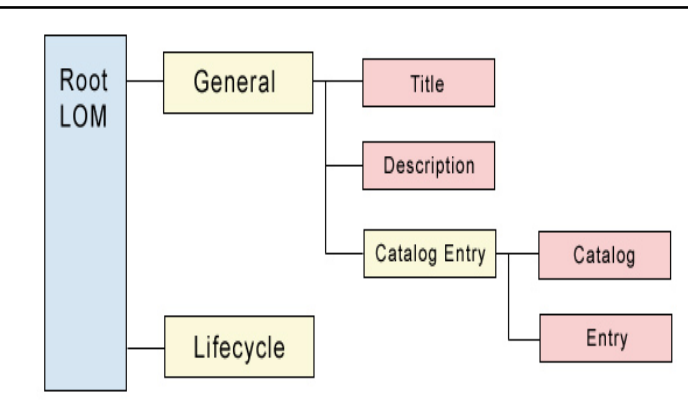

Figure 1: Hierarchical view of metadata elements

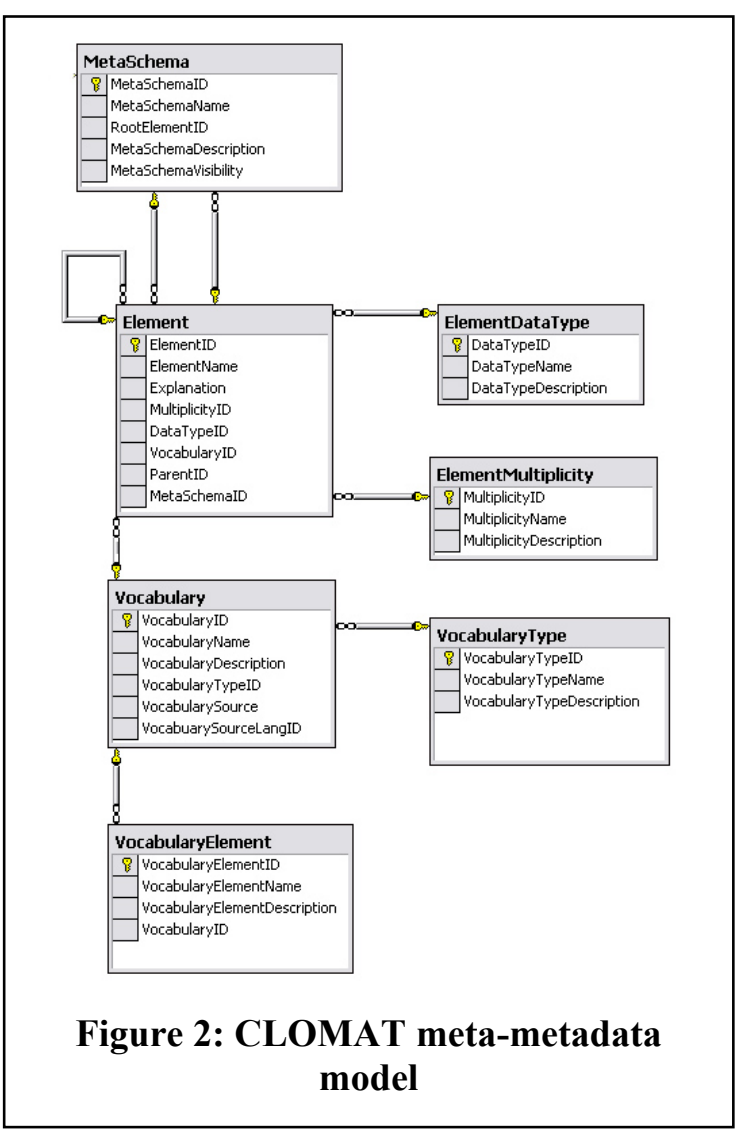
meta-metadata models as means of standardizing the types of metadata used and tracked by distinct functions that those metadata elements serve.

Based on the LOM model, the metadata model contains the following necessary pieces of information as they are used to describe the metadata elements:

- Number: Hierarchical number within the metadata model

- Name: Element name

- Explanation: Detailed description of the element

- Multiplicity: the number of instances of the element that are allowed. Possible values are: one and only one; one or more; zero or one; zero or more. 
- Data Type: Whether the element's value is textual, numerical or a date, and any constraints on its size and format. There are four general-purpose types used in the LOM information model: string type, langstring type, date type, and vocabulary type. A string represents a string of characters. A langstring represents a string of characters which has associated a human language as defined by ISO 639 standard (2005). Langstrings are a good way to support multi-language environments. Date represents a date or time expressed as an ISO 8601 standard (2005) and a description of the date/time. Vocabulary represents a source for langstring items. For those elements that have a data type of a vocabulary type, additional information is provided on whether or not the vocabulary is a restricted or best practice vocabulary. Restricted indicates that the metadata element is restricted to the vocabulary entries listed. Best practice indicates that the LOM recommends, as best practice, to use the vocabulary entries listed.

Studying in detail the type of metadata elements that are part of a metadata model, it is very easy to find generalizations due to the general-purpose types that restrict the model. For example, the 'entity' element that is used to describe information about the people and organizations in the SCORM model is defined as being of string type, but there is a note saying that this element should be a vCard (2005). The implications of such a definition replicate into the flexibility of the model and the tool that implements the model. Since the element is defined as being of string type, the element would have associated the behavior of that particular type. The extra behavior that has to be associated with a vCard would have to be added through additional code for that individual element. Coding the behavior for each element of a model means to hard code the model within the tool. Therefore, if we want to represent different models within the same tool, we need to start from defining the metadata types. We want to represent different models within the same tool.

The idea of dynamic tools that represent flexible models has to be backed up by well-defined meta-metadata model. The CLOMAT prototype reflects this philosophy. Rather than starting with the individual metadata elements from the LOM, or any model, and trying to represent them within the tool design, it starts from the relevant metadata element type and their behavior. Thus, CLOMAT addresses specific functions for different types of metadata and establishes generic types as a means of regulating the metadata types that should be tracked throughout these functions, regardless of the metadata elements defined as being of those types. The metadata elements will be just instances of those metadata types inheriting their behavior. Table 1 represents the three-layer architecture of CLOMAT.

Table 1: Three-Layer Architecture

\begin{tabular}{|l|l|l|}
\hline LAYER & \multicolumn{1}{|c|}{ DESCRIPTION } \\
\hline M3 & Meta-metadata model & $\begin{array}{l}\text { It contains the rules and specifications for the representation of any } \\
\text { hierarchical structure describing a metadata model and the imple- } \\
\text { mentation of metadata types with their associated behavior. }\end{array}$ \\
\hline M2 & Metadata model & $\begin{array}{l}\text { It is an instance of meta-metadata model. The flexibility and exten- } \\
\text { sibility of M2 metadata model come from the underlining M3 layer } \\
\text { properties which allow for an easy representation of M2 based on } \\
\text { the previously defined metadata types and for rapid changes to the } \\
\text { hierarchical structure based on the rules and specifications previ- } \\
\text { ously defined. }\end{array}$ \\
\hline M1 & Data model & $\begin{array}{l}\text { It is an instance of metadata model. It represents the user data from } \\
\text { digital objects templates and records where the valid structure of } \\
\text { such data is defined at the M2 level. }\end{array}$ \\
\hline
\end{tabular}


Layer M3, the meta-metadata model, allows for representation of flexible M2 metadata models by letting individually defined metadata elements of different types to be arranged in a desired hierarchical structure. Thus, the M2 Metadata model becomes an instance of M3 meta-metadata model with elements that mimic the behavior of their specified types. Thus, the meta-metadata model provides a rigorous infrastructure at a higher level of abstraction and furnishes an architectural basis for extension of metadata models.

\section{Processing Metadata at Run-Time}

The metadata types designed in CLOMAT are intended to prove the concept of how changing the focus from creating metadata models with many elements to creating meta-metadata models can provide a more efficient and more flexible, design for metadata creation tools.

\section{Prototype Features}

The user group for this metadata authoring tool is not intended to be an exclusive group of content creators such as librarians, instructional designers or information professionals familiar with metadata standards; it is for a broad audience including any metadata creator. The features outlined below are supported by an intuitive, informative, engaging, easy to navigate web interface. Relevancy and clarity of the information, adequate feedback, reduced workload, and most importantly, overall consistency and simplicity were the principles that drove the implementation of CLOMAT. Based on the design issues above and the framework previously discussed, the CLOMAT prototype brings together the following aspects of design.

\section{Metadata Model Flexibility}

Due to the metadata type-driven design, CLOMAT provides the option of defining multiple metadata models, and all of them are available at the same time for use (see Figure 3). Figure 4 represents the trees corresponding to the SCORM model (an adaptation of LOM) and to "My metadata model," a custom model which has been defined according to the metametadata model outlined above.

\section{CLOMAT}

Customizable Learning Objects Metadata Authoring Tool

Metadata Information Model Manager | Metadata Menu | Logout

Valentina's Profile

Create a model view

create a model template

Define a new model

Figure 3: CLOMAT metadata models flexibility a) Select a metadata information model for which you would like to create a custom template.

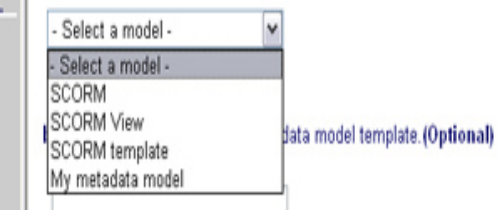

Step 1 of 2: Select a model for the template

-
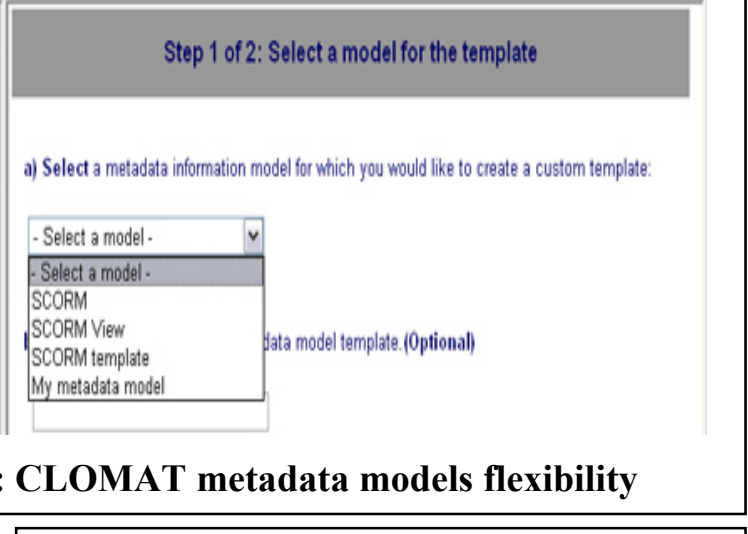


\section{User Management}

User profiles facilitate the automation of those metadata elements related to the content creator. They also allow for customization of the metadata model within each individual profile. Metadata model views and metadata model templates provide a great degree of flexibility for users to personalize the metadata models according to their needs and expertise. The interface provides simple mechanisms (e.g. checkboxes for hiding/revealing metadata elements from schemas or dropdown lists for changing the type, the multiplicity, or the vocabulary of a metadata element). This version of the CLOMAT prototype does not include the full collaborative aspect envisioned in the framework, but it does have some collaborative features such as metadata model sharing and vCard exchange.

\section{Tree-Based Representation}

A tree-based structure is used to represent the internal database structure and allows the users to navigate the metadata. An event-based engine parses the events related to metadata elements once the user clicks the element in the tree structure and permits their processing at run-time.

\section{External Standards Support}

External Standards, such as vCard standard (1996), MIME standard (1996), URI standard ("Naming and addressing", 1993), ISO 639 standard (2005) for language codes, ISO 8601 standard (1997) for dates and times, are incorporated into the prototype in such a way that a non-metadata expert can make use of them. To achieve interoperability with other tools, applications, and repositories, an import/export XML document feature is provided.

\section{Multiple Language Support}

Every element of type langstring has a language associated with it. A user can set the default language and then assign it to each langstring element to edit. The user can provide the most important elements in several languages to reach a larger audience by duplicating langstrings within an element. If a language is not specified, no language will be associated with the langstring elements.

\section{Custom View Accessibility}

Users will likely not want to view the entire metadata information model to get to the few pieces of metadata of interest. For this reason, CLOMAT offers the possibility of defining custom views of metadata models. This feature can be used to facilitate collaboration by allowing the users who are responsible for different parts of the metadata for the same learning object to have their own custom view of that metadata.

\section{Template-Driven Editing}

A metadata template is an aid to help users create metadata faster by not editing the same information twice. The template-driven editing, along with the metadata model views, was facilitated by the three-layer architecture of the design model of CLOMAT. Both model templates and model views are instances of the meta-metadata model. When an author starts working on a learning object metadata using a template, the record for that learning object is pre-filled with existing data from the template. In a collaborative environment, a metadata template can be exchanged among users. CLOMAT enables users to have several templates and custom views at the same time. Each template can be based on different metadata models or model views of models according to the needs of the users (see Figure 5). 


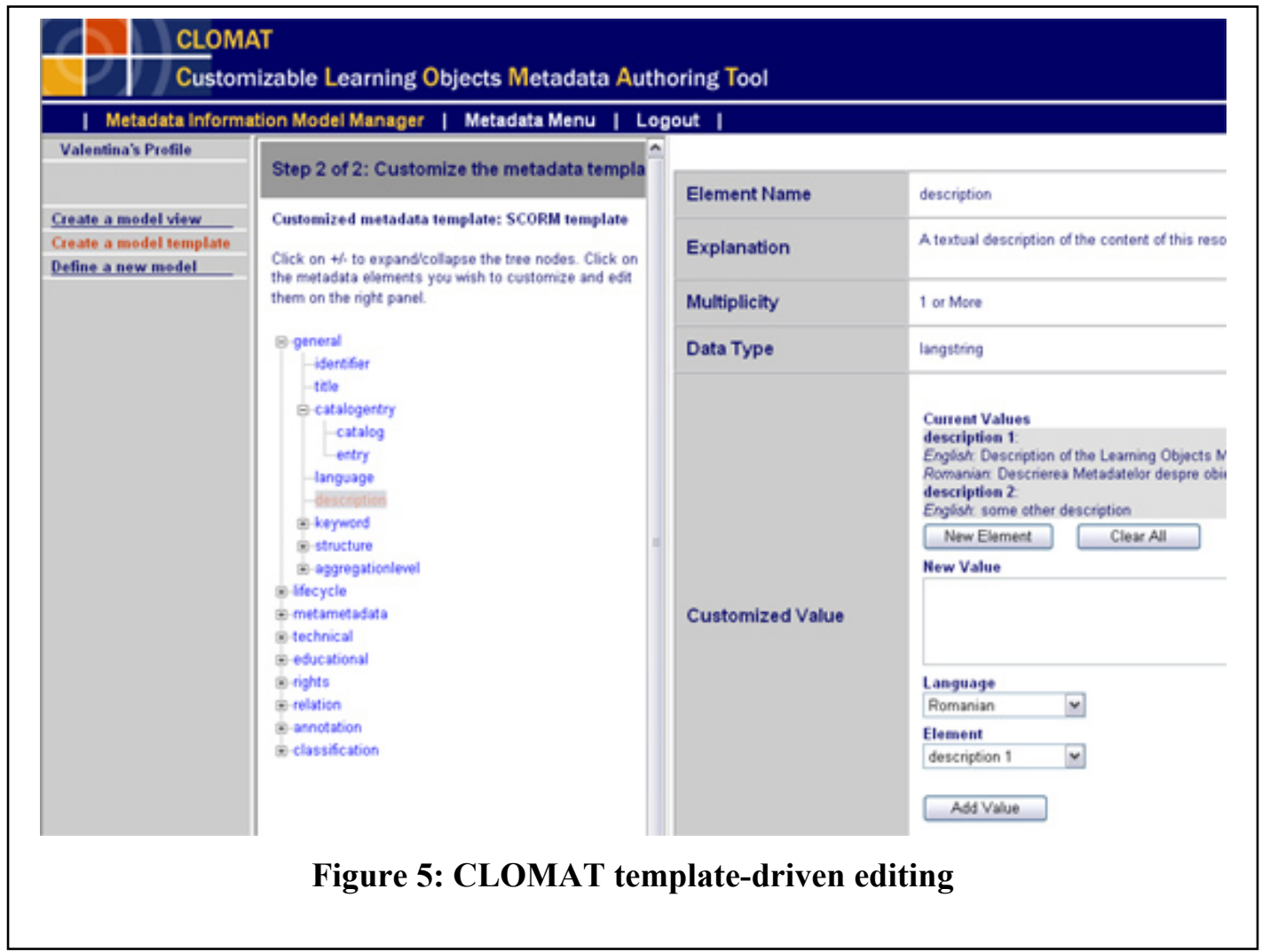

\section{Contextual Help}

The contextual help supports interaction between content creators and the CLOMAT prototype in the following ways.

- Immediate assistance without their having to leave the context in which they are working

- Information about a particular metadata attribute, its context, and its corresponding vocabulary

- Answers to questions such as "What is this?" and "Why would I use it?"

\section{Exploratory Study of CLOMAT}

The objective of this exploratory study is to determine the extent to which the CLOMAT prototype can be used to achieve the specified goal: editing learning object metadata records with effectiveness, efficiency, and user satisfaction. The evaluation of the prototype tested its functionality and usability according to predefined criteria and against the principles that led to its development. The following characteristics were specifically addressed.

- How easy the tool is to learn. After an introduction one day before the test, could users create their own accounts and efficiently interact with the interface?

- The degree to which the tool supports the tasks the user wishes to perform and how the user understands them.

- How much time is saved by using templates to create metadata for the learning objects.

These characteristics were considered as the criteria during evaluation. Five participants with no previous experience in editing metadata, but with general domain knowledge, were selected for this usability test using three scenarios of different degrees of complexity. The first task was to 
define a metadata standard in CLOMAT. The second task was to enter metadata into CLOMAT. The third task was to enter metadata into CLOMAT using a pre-defined template. Performance on these three tasks was compared with entering metadata into a traditional editor. Task times were recorded to measure any efficiency gain that CLOMAT might have. The exploratory study resulted in the following findings:

- Time savings by using metadata templates. The average time for editing a complete metadata record was 25 minutes in a traditional editor. The average time for editing the reduced number of metadata elements was 4 minutes. The savings in terms of time were, on average, 20 minutes per learning object, which for a course with 2500 learning objects translates to 50,000 minutes (approximately 833 hours).

- The contextual help assisted in defining the metadata. All subjects made use of the contextual help while defining the metadata. The help information was derived from the standards documents. The terminology used sometimes proved too technical for the users, and it did not often clarify what the metadata element referred to. It is therefore difficult to quantify the impact of contextual help on improving the entry tasks.

- The tabular presentation may be more efficient than the tree structure. The tabular structure seemed to be understood by the users quicker than the tree structure. Also, the tree structure sometimes had too much information attached to it, making it confusing. For example, users had to click on $+/$ - to extend the nodes. They had to click on the name of the element to display information about it. And they had to click on the checkbox attached to element to select/deselect the element.

- Advanced concepts may require advanced methods of illustration. The relationship between objects, an advanced concept that is very important for reuse, was not understood by users without detailed explanation. It may be that more visual representations of such relations will facilitate a better understanding. Further investigation is necessary regarding this concept. A University of North Carolina study supports this finding, showing that users spent most of their time focused on only a few subtasks (defined as time spent harvesting and entering information for a specific element), and defining the relation between learning objects took $23 \%$ of their total effort.

- Metadata quality. The quality of metadata was influenced by the user's interpretation of the metadata elements and their values. The meaning of some of these elements was not immediately obvious to users and, as noted above, help based on the standards documentation is not sufficient. These misinterpretations led to some meaningless metadata. Some users entered the keywords one by one, while others input all the keywords in one element. This issue would have proved significant when it came to searching for the learning objects in a collection.

\section{Conclusions}

In the Knowledge Age, there is an obvious requirement for metadata as a means for exchange, discovery, and sharing of digital objects. Metadata is used by the applications, repositories, content creators, and system developers. In environments in which there are potentially thousands of objects available, the metadata must be cost-effective, accurate, and complete. The CLOMAT prototype demonstrated several techniques to overcome some of the main difficulties of this domain, such as editing the metadata for a large number of learning objects or responding to users' needs for customization of metadata models and organization of the information presented. The prototype is a step toward a user-centered future in which everyone can be as comfortable editing metadata as they are editing text today. The CLOMAT prototype was proved to have economical value by reducing the time of editing metadata. However, the experience with the CLOMAT pro- 
totype illustrates that metadata creation for learning objects is a complex issue, and developing a tool for a particular metadata standard does not solve all difficulties.

There are other process-related challenges and organization-specific requirements that a metadata solution has to address. This study has identified challenges related to users' needs, metadata standards, and learning environment and proposed a solutions to these challenges. Users require better interfaces to support their goals and better guidance than that available in standards documentation.

During this research, collaborative editing was identified as being one of the critical features in achieving quality metadata. Learning objects metadata, with its different functions represents an appropriate subject for collaborative editing. For example, the design specification for a new learning object can be provided by the subject matter expert. The instructional designer can edit those metadata elements related to pedagogy. And the technical staff can take care of technical details such as the software needed to access the learning resource or the size in bytes of the resource. In this way, metadata can reach a higher level of richness, completeness, and accuracy.

\section{References}

Arms, W. Y., Hillmann, D., Lagoze, C., Krafft, D., Marisa, R., Saylor, J., Terrizzi, C., \& Van de Sompel, H. (2002). A Spectrum of interoperability: The site for science prototype for the NSDL. D-Lib Magazine, 8(1), Retrieved August 26th, 2005, from http://www.dlib.org/dlib/january02/arms/01arms.html

Barker, E., \& Ryan, B. (2005). Case studies in implementing educational metadata standards. Centre for Educational Technology Interoperability Standards, United Kingdom. Retrieved August 26th, 2005, from http://metadata.cetis.ac.uk/usage survey/

Candan, K. S., Liu, H., \& Suvarna, R. (2001). Resource description framework: Metadata and its applications. ACM Knowledge, Discovery and Data Mining Explorations, 3 (1), 6 - 19.

Dublin Core Metadata Abstract Model. (2005) Retrieved August 26th, 2005, from http://dublincore.org/documents/abstract-model/

Dumais S. T., \& Chen H. (2000). Hierarchical classification of web content. Proceedings of the 23rd Int'l ACM Conf. on Research and Development in Information Retrieval (SIGIR), Athens, Greece, 256-263.

Garcia, F. (2000). CACTUS: Automated tutorial course generation for software applications. Proceedings of the $5^{\text {th }}$ International Conference on Intelligent User Interfaces, New Orleans, Louisiana, 113-120.

Geisler, G., McArthur, D., \& McClelland, M. (2002). Creating virtual collections in digital libraries: Benefits and implementation issues. Joint Conference on Digital Libraries, Portland, Oregon, 210-218.

Greer, L. R. (2002). The learning matrix: Cataloging resources with rich metadata. Proceedings of the IEEE-CS Joint Conference on Digital Libraries (Portland, Oregon, JCDL 2002), 375.

Grissom, S., Knox, D., Copperman, E., Dann, W., Goldweber, M., Hartman, J., Kuittinen, M., Mutchler, D., \& Parlante, N. (1998). Developing a digital library of computer science teaching resources. Proceedings of the Annual Joint Conference Integrating Technology into Computer Science Education, Dublin, Ireland, 1-13.

ISO 639 - International standard on language codes. (2005). Library of Congress. Retrieved August 26th, 2005, from http://www.loc.gov/standards/iso639-2/iso639jac.html

ISO 8601 - International standard for the representation of dates and times. (1997) World Wide Web Consortium. Retrieved August 26th, 2005, from http://www.w3.org/TR/NOTE-datetime

Kassanke, S., \& Steinacker, A. (2000). Learning objects metadata and tools in the area of operations research. IEEE Learning Technology Journal, 5(1), Retrieved August 26th, 2005, from http://ttf.ieee.org/learn_tech/issues/january2003/

Kunze, T., Brase, J., \& Nejdl, W. (2002). Editing learning object metadata: Schema driven input of RDF metadata with the OLR3-Editor. Semantic authoring, annotation \& knowledge markup workshop. Proceedings of the 15th European Conf. on Artificial Intelligence, Lyon, France, July, 22-26. 
Lagoze, C., \& Van de Sompel, H. (2001).The open archives initiative protocol for metadata harvesting, Open Archives Initiative, January, Retrieved August 26th, 2005, from http://www.openarchives.org/OAI/openarchivesprotocol.htm

Learning Objects Metadata [LOM]. (2002). IEEE Learning Technology Standardization Committee. Retrieved August 26th, 2005, from http://ltsc.ieee.org/

Marshall, C. C. (1998). Making metadata: A study of metadata creation for a mixed physical-digital collection. Proceedings of the 3rd ACM Conference on Digital Libraries, (Pittsburgh, Pennsylvania, June, 1998) 162-171.

MIME: Multipurpose Internet Mail Extensions. (1996). IETF - Internet Engineering Task Force, Network Working Group. Retrieved August 26th, 2005, from http://www.ietf.org/rfc/rfc2046.txt

Naming and addressing: URIs, URLs... (1993). World Wide Web Consortium. Retrieved August 26th, 2005, from www.w3.org/Addressing/

Poyry, P., Pelto-Aho, K., \& Puustjarvi, J. (2002). The role of metadata in the CUBER system. Proceedings of the South African Institute of Computer Scientists and Information Technologists on Enablement through Technology, (Port Elizabeth, South Africa, 2002), 172-178.

Ryu, J., Sohn, Y., \& Kim, M. (2002). MPEG-7 metadata authoring tool. Proceedings of the tenth ACM multimedia Conference, Juan-les-Pins, France.

Saddik, E. A., Ghavam, A., Fischer, S., \& Steinmetz, R., (2000). Metadata for smart multimedia learning objects. Proceedings of the fourth Australasian Computing Education Conference (Melbourne, Australia, December, 87-94.

South, J. B., \& Monson, D. W. (2000). A university-wide system for creating, capturing, and delivering learning objects. In D. A. Wiley (Ed.), The instructional use of learning objects. Retrieved August 26th, 2005, from http://reusability.org/read/chapters/south.doc

vCard. The Electronic Business Card Version 2.1. (1996). A versit consortium specification. Retrieved August 26th, 2005, from www.imc.org/pdi/vcard-21.doc

Yao, A., \& Jin, J. (2001). The development of a video metadata authoring and browsing system in XML. Proceedings of the International Conference on Visualization, Sydney, Australia, 39-46.

\section{Biographies}
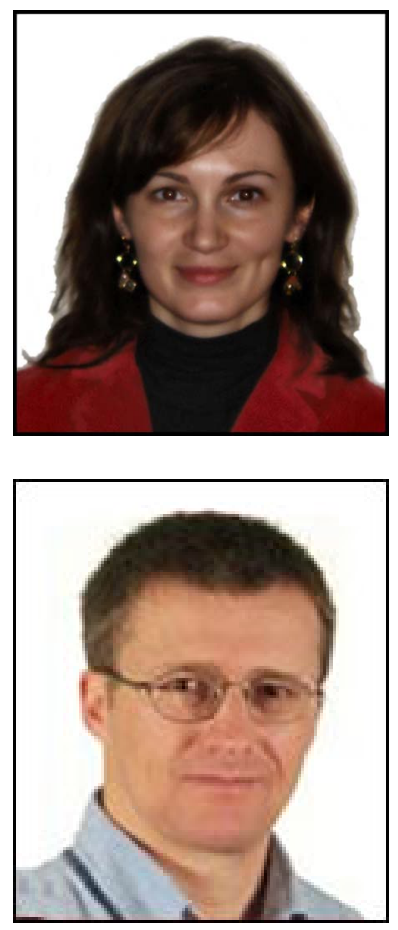

Valentina Malaxa has a Masters in Computer Science from Florida State University. She worked for four years at the Learning Systems Institute where she developed interests in learning objects, metadata standards, and human-computer interaction. She currently works as a Software Engineer at DuvaSawko where she designs electronic data interchange software systems for the healthcare billing chain.

Ian Douglas, Ph. D. has a joint appointment as an assistant professor with the Learning Systems Institute (LSI) and College of Information at Florida State University. He leads LSI's Knowledge Communities Research Group (http://www.lpg.fsu.edu/kcrg/) and is involved in a number of research initiatives related to the US military's Advanced Distributed Learning Initiative. Dr. Douglas's general interests are in human factors in technology, general systems thinking, organizational learning and knowledge management. He has previously conducted research in artificial intelligence and simulation-based training systems. 